\title{
INEQUALITIES FOR ALGEBRAIC POLYNOMIALS ON AN ELLIPSE ${ }^{1}$
}

\author{
Tatiana M. Nikiforova \\ Krasovskii Institute of Mathematics and Mechanics, \\ Ural Branch of the Russian Academy of Sciences, \\ 16 S. Kovalevskaya Str., Ekaterinburg, 620990, Russia \\ Ural Federal University, \\ 19 Mira str., Ekaterinburg, 620002, Russia \\ t.m.nikiforova@yandex.ru
}

\begin{abstract}
The paper presents new solutions to two classical problems of approximation theory. The first problem is to find the polynomial that deviates least from zero on an ellipse. The second one is to find the exact upper bound of the uniform norm on an ellipse with foci \pm 1 of the derivative of an algebraic polynomial with real coefficients normalized on the segment $[-1,1]$.
\end{abstract}

Keywords: Polynomial, Chebyshev polynomials, Ellipse, Segment, Derivative of a polynomial, Uniform norm.

\section{Introduction}

Denote by $\mathcal{P}_{n}^{1}$ the set of algebraic polynomials of degree $n$ with the unit leading coefficient:

$$
p(z)=z^{n}+c_{n-1} z^{n-1}+\ldots+c_{0}, \quad c_{k} \in \mathbb{C} .
$$

Consider the ellipse $E=\{z=a \cos t+i b \sin t \mid t \in[0,2 \pi] ; a>b>0\}$ centered at the origin. Let $\|p\|_{E}=\max _{z \in E}|p(z)|$. Recall that a polynomial $p_{n}^{*} \in \mathcal{P}_{n}^{1}$ is called a polynomial least deviating from zero on $E($ in $C(E))$ if

$$
\min _{p \in \mathcal{P}_{n}^{1}}\|p\|_{E}=\left\|p_{n}^{*}\right\|_{E}
$$

V.I. Smirnov and N.A. Lebedev showed in 1964 [1, p. 331-333] that the normalized Chebyshev polynomial of the first kind $T_{n}$ is the polynomial least deviating from zero on an ellipse with foci \pm 1 . Smirnov and Lebedev considered the ellipse as an image of a circle under the Joukowsky transform

$$
f(\omega)=(\omega+1 / \omega) / 2 .
$$

Thus, instead of polynomials, they studied functions of the following form defined on circles:

$$
q_{n}(\omega)=R_{n}(\omega)+\sqrt{\omega^{2}-1} \cdot Q_{n-1}(\omega),
$$

where $R_{n}(\omega)$ and $Q_{n-1}(\omega)$ are polynomials of degree at most $n$ and $n-1$, respectively. We will give another solution of this problem. Our proof is based on Kolmogorov's theorem about the best uniform approximation of arbitrary continuous function by generalized polynomials.

\footnotetext{
${ }^{1}$ The work was performed as a part of research conducted in the Ural Mathematical Center.
} 
The second problem considered in the paper is related to inequalities for derivatives of algebraic polynomials. Let

$$
E_{1}=\left\{z=a \cos t+b i \sin t \mid t \in[0,2 \pi] ; a^{2}-b^{2}=1\right\}
$$

be an ellipse with foci \pm 1 , and let $\mathcal{P}_{n}^{[-1,1]}$ be the set of algebraic polynomials of degree $n$ with real coefficients and the unit uniform norm on $[-1,1]$. In 1986, J.H.B. Kemperman showed [3, Theorem 1.2] that, for any $p_{n} \in \mathcal{P}_{n}^{[-1,1]}$,

$$
\left\|p_{n}^{\prime}\right\|_{E_{1}} \leq \frac{n}{2 b}\left((a+b)^{n}-(a-b)^{n}\right)=\left\|T_{n}^{\prime}\right\|_{E_{1}}
$$

We obtained the proof of this fact independently of Kemperman. Our idea and Kemperman's one are similar, but our proof is shorter and easier to understand, as we consider objects in the problem from a slightly different point of view.

\section{Polynomial that deviates least from zero on an ellipse}

Let us give a solution to the problem about a polynomial $p_{n}^{*}(z)$ deviating least from zero on $E$. We will obtain an explicit formula for $p_{n}^{*}(z)$, a value of its norm $\left\|p_{n}^{*}\right\|_{E}$, and a recurrence relation between $p_{n+1}^{*}(z), p_{n}^{*}(z)$, and $p_{n-1}^{*}(z)$.

Lemma 1. For any positive integer $n$,

$$
(a \cos t+i b \sin t)^{n}=A_{n} \cos (n t)+i B_{n} \sin (n t)+\sum_{k=0}^{n-1} \alpha_{k} \cos (k t)+i \beta_{k} \sin (k t),
$$

where $A_{n}, B_{n}, \alpha_{k}, \beta_{k} \in \mathbb{R}$ and

$$
A_{n}=\frac{a A_{n-1}+b B_{n-1}}{2}=\frac{(a+b)^{n}+(a-b)^{n}}{2^{n}}, \quad B_{n}=\frac{b A_{n-1}+a B_{n-1}}{2}=\frac{(a+b)^{n}-(a-b)^{n}}{2^{n}} .
$$

P r o o f. The proof of (1.1) is by induction on $n$. It is clear that the statement holds for $n=1$. Assume that the statement holds for $n-1$, and prove it for $n$. We have

$$
\begin{gathered}
(a \cos t+i b \sin t)^{n}=(a \cos t+i b \sin t) \cdot\left(A_{n-1} \cos ((n-1) t)+i B_{n-1} \sin ((n-1) t)+\right. \\
\left.+\sum_{k=0}^{n-2} \widetilde{\alpha}_{k} \cos (k t)+i \widetilde{\beta}_{k} \sin (k t)\right)
\end{gathered}
$$

with real $A_{n-1}, B_{n-1}, \widetilde{\alpha}_{k}$, and $\widetilde{\beta}_{k}$. The imaginary unit will only appear in the products of the form

$$
i \sin (\ell t) \cos (k t)=i(\sin ((k+\ell) t)-\sin ((k-\ell) t)) / 2 .
$$

Therefore, all the coefficients $A_{n}, B_{n}, \alpha_{k}$, and $\beta_{k}$ in (1.1) are real.

The proof of the recurrence relations for $A_{n}$ and $B_{n}$ is straightforward. Indeed, by removing parentheses in the product, we obtain

$$
\begin{gathered}
(a \cos t+i b \sin t) \cdot\left(A_{n-1} \cos ((n-1) t)+i B_{n-1} \sin ((n-1) t)\right)= \\
=\frac{a A_{n-1}+b B_{n-1}}{2} \cos (n t)+\frac{b A_{n-1}+a B_{n-1}}{2} i \sin (n t)+\ldots
\end{gathered}
$$


Let us show that

$$
A_{n}-B_{n}=\frac{(a-b)^{n}}{2^{n-1}}, \quad A_{n}+B_{n}=\frac{(a+b)^{n}}{2^{n-1}}, \quad n \in \mathbb{N} .
$$

These equations obviously imply explicit formulas for $A_{n}$ and $B_{n}$. Equations (1.2) for $n=1$ are obvious. We now proceed by induction. Assume that the statement holds for $n-1$, and prove that it holds for $n$. We have

$$
\begin{gathered}
A_{n}+B_{n}=\frac{a A_{n-1}+b B_{n-1}}{2}+\frac{b A_{n-1}+a B_{n-1}}{2}= \\
=\frac{1}{2}\left((a+b) A_{n-1}+(a+b) B_{n-1}\right)=\frac{(a+b)}{2} \cdot \frac{(a+b)^{n-1}}{2^{n-2}}=\frac{(a+b)^{n}}{2^{n-1} .}
\end{gathered}
$$

Similarly, we obtain the formula for $A_{n}-B_{n}$.

Corollary 1. For any $p \in \mathcal{P}_{n}^{1}(\mathbb{C})$, its restriction to the ellipse $E$ is represented as

$$
p(z)=A_{n} \cos (n t)+i B_{n} \sin (n t)+\sum_{k=0}^{n-1} \alpha_{k} \cos (k t)+\beta_{k} \sin (k t),
$$

where $\alpha_{k}, \beta_{k} \in \mathbb{C}, A_{n}, B_{n} \in \mathbb{R}$ and $A_{n} \geq B_{n}$.

We will not change the meaning of symbols $A_{n}$ and $B_{n}$.

Lemma 2. Let $\varphi=\left\{1,(a \cos t+i b \sin t), \ldots,(a \cos t+i b \sin t)^{n-1}\right\}$, and let $\Phi_{n-1}$ be the set of generalized polynomials from $\varphi$ with complex coefficients. Then, for $f(t)=A_{n} \cos (n t)+i B_{n} \sin (n t)$,

$$
\inf _{p \in \Phi_{n-1}}\|f-p\|_{C[0,2 \pi]}=\|f\|_{C[0,2 \pi]}=A_{n}
$$

i.e., $p(t) \equiv 0$ is the best uniform approximation for $f$ by generalized polynomials from $\varphi$.

P r o o f. Let $\varepsilon=\left\{t \in[0,2 \pi]:|f(t)|=\|f\|_{C[0,2 \pi]}=A_{n}\right\}$. Since the set of values of $f$ is the ellipse with the major horizontal semiaxis of length $A_{n}$ and the vertical semiaxis of length $B_{n}$, we have

$$
\varepsilon=\left\{t_{k}=\pi k / n, k=0, \ldots, 2 n\right\} .
$$

By Kolmogorov's theorem [2, Theorem 1; 8, p. 47, Theorem 1], to prove the lemma it suffices to show that

$$
\min _{t_{k}} \Re\left\{p\left(t_{k}\right) \overline{f\left(t_{k}\right)}\right\} \leq 0
$$

for any $p \in \Phi_{n-1}$. Let us substitute $f\left(t_{k}\right)$ into (1.3):

$$
\Re\left\{p\left(t_{k}\right) \overline{f\left(t_{k}\right)}\right\}=A_{n} \Re\left\{(-1)^{k} p\left(t_{k}\right)\right\}, \quad k=0, \ldots, 2 n .
$$

Note that, by the definition of $\varphi$, it follows that $p(t)$ is a trigonometric polynomial of degree $n-1$. If $p\left(t_{k}\right)$ vanishes at least at one point $t_{k}$, then inequality (1.3) holds.

Assume that

$$
\min _{t_{k}}\left\{(-1)^{k} p\left(t_{k}\right)\right\}>0, \quad k=0, \ldots, 2 n .
$$

From our assumption, it follows that $p(t)$ takes values of different signs at $2 n+1$ points on $[0,2 \pi]$. Since $p(t)$ is a continuous function, it has at least $2 n$ zeros on $[0,2 \pi]$. But this is impossible, as any trigonometric polynomial of degree $n-1$ has at most $2(n-1)$ zeros on $[0,2 \pi)$. Therefore,

$$
\min _{t_{k}}\left\{(-1)^{k} p\left(t_{k}\right)\right\} \leq 0, \quad k=0, \ldots, 2 n .
$$

The statement is proved. 
Theorem 1. Consider the ellipse $E=\{z=a \cos t+i b \sin t, t \in[0,2 \pi]\}, a>b>0$. The following polynomial deviates least from zero on $\mathrm{E}$ :

$$
p_{n}^{*}(z)=\frac{1}{2^{n-1}} \sum_{k=0}^{[n / 2]} C_{n}^{2 k} z^{n-2 k}\left(z^{2}-c^{2}\right)^{k}=\frac{1}{2^{n}}\left(\left(z+\sqrt{z^{2}-c^{2}}\right)^{n}+\left(z-\sqrt{z^{2}-c^{2}}\right)^{n}\right),
$$

where $c^{2}=a^{2}-b^{2}$. Moreover, $\left\|p_{n}^{*}\right\|_{C(E)}=\frac{(a+b)^{n}+(a-b)^{n}}{2^{n}}$ and the following recurrence relation holds:

$$
p_{n+1}^{*}(z)=z p_{n}^{*}(z)-\frac{c^{2}}{4} p_{n-1}^{*}(z) .
$$

$\mathrm{P}$ r o o f. Let us show that $A_{n} \cos (n t)+i B_{n} \sin (n t)$ is the restriction to $E$ of an algebraic polynomial from $\mathcal{P}_{n}^{1}$. Lemma 2 implies that this polynomial is a solution to the problem

$$
\begin{gathered}
A_{n} \cos (n t)+i B_{n} \sin (n t)=\frac{A_{n}}{2}\left(e^{i n t}+e^{-i n t}\right)+\frac{i B_{n}}{2 i}\left(e^{i n t}-e^{-i n t}\right)= \\
=\frac{A_{n}}{2}\left[(\cos t+i \sin t)^{n}+(\cos t-i \sin t)^{n}\right]+\frac{B_{n}}{2}\left[(\cos t+i \sin t)^{n}-(\cos t-i \sin t)^{n}\right]= \\
=\frac{(a+b)^{n}}{2^{n}}(\cos t+i \sin t)^{n}+\frac{(a-b)^{n}}{2^{n}}(\cos t-i \sin t)^{n}= \\
=\frac{1}{2^{n}}\left([(a \cos t+b i \sin t)+(b \cos t+a i \sin t)]^{n}+[(a \cos t+b i \sin t)-(b \cos t+a i \sin t)]^{n}\right) .
\end{gathered}
$$

Since

$$
z^{2}=a^{2} \cos ^{2} t+2 a b i \cos t \sin t-b^{2} \sin ^{2} t=b^{2} \cos ^{2} t+2 a b i \sin t \cos t-a^{2} \sin ^{2} t+\left(a^{2}-b^{2}\right),
$$

we have

$$
(b \cos t+a i \sin t)^{2}=z^{2}-\left(a^{2}-b^{2}\right) .
$$

Hence,

$$
\begin{gathered}
A_{n} \cos (n t)+i B_{n} \sin (n t)= \\
=\frac{1}{2^{n}}\left\{\sum_{k=0}^{n} C_{n}^{k} z^{n-k}\left(z^{2}-\left(a^{2}-b^{2}\right)\right)^{k / 2}+\sum_{k=0}^{n} C_{n}^{k} z^{n-k}(-1)^{k}\left(z^{2}-\left(a^{2}-b^{2}\right)\right)^{k / 2}\right\}= \\
=\frac{1}{2^{n}} \sum_{0 \leq k \leq n, k / 2 \in \mathbb{Z}} 2 C_{n}^{k} z^{n-k}\left(z^{2}-\left(a^{2}-b^{2}\right)\right)^{k / 2}=\frac{1}{2^{n-1}} \sum_{0 \leq k \leq n, k / 2 \in \mathbb{Z}} C_{n}^{k} z^{n-k}\left(z^{2}-\left(a^{2}-b^{2}\right)\right)^{k / 2} .
\end{gathered}
$$

It is obvious that

$$
\left\|p_{n}^{*}\right\|_{C(E)}=A_{n}=\frac{(a+b)^{n}+(a-b)^{n}}{2^{n}} .
$$

Let us prove the recurrence relation. We have

$$
\begin{gathered}
(a \cos t+i b \sin t)\left(A_{n} \cos (n t)+i B_{n} \sin (n t)\right)-\frac{\left(a^{2}-b^{2}\right)}{4}\left[A_{n-1} \cos ((n-1) t)+i B_{n-1} \sin ((n-1) t)\right]= \\
=\frac{a A_{n}+b B_{n}}{2} \cos ((n+1) t)+\frac{b A_{n}+a B_{n}}{2} i \sin ((n+1) t)+ \\
+\left(\frac{a A_{n}-b B_{n}}{2}-\frac{\left(a^{2}-b^{2}\right) A_{n-1}}{4}\right) \cos ((n-1) t)+\left(\frac{a B_{n}-b A_{n}}{2}-\frac{\left(a^{2}-b^{2}\right) B_{n-1}}{4}\right) i \sin ((n-1) t) .
\end{gathered}
$$


It can be easily checked that the coefficients at $\cos ((n-1) t)$ and $\sin ((n-1) t)$ are equal to zero:

$$
\frac{a A_{n}-b B_{n}}{2}-\frac{\left(a^{2}-b^{2}\right) A_{n-1}}{4}=\frac{a\left(a A_{n-1}+b B_{n-1}\right)-b\left(b A_{n-1}+a B_{n-1}\right)-A_{n-1}\left(a^{2}-b^{2}\right)}{4}=0 .
$$

As above, the coefficient at $\sin ((n-1) t)$ is equal to zero. The recurrence relation is proved.

Note that $p_{n}^{*}(z)=z^{n}$ for $a=b=1$ and $p_{n}^{*}(z)=T_{n}(z) / 2^{n-1}$ for $a=1$ and $b=0$. It corresponds to well-known results for the unit circle and the interval $[-1,1]$.

\section{Inequality for the derivative of an algebraic polynomial with real coefficients on an ellipse}

Let us give a solution to the problem of finding the best constant $M$ in the inequality

$$
\left\|p_{n}^{\prime}\right\|_{E_{1}} \leq M, \quad p_{n} \in \mathcal{P}_{n}^{[-1,1]}
$$

Theorem 2. The following inequality holds for every polynomial $p_{n} \in \mathcal{P}_{n}^{[-1,1]}$ :

$$
\left\|p_{n}^{\prime}\right\|_{E_{1}} \leq \frac{n}{2 b}\left[(a+b)^{n}-(a-b)^{n}\right] .
$$

The Chebyshev polynomial of the first kind

$$
T_{n}(z)=\sum_{k=0}^{[n / 2]} C_{n}^{2 k} z^{n-2 k}\left(z^{2}-1\right)^{k}=\frac{1}{2}\left(\left(z+\sqrt{z^{2}-1}\right)^{n}+\left(z-\sqrt{z^{2}-1}\right)^{n}\right)
$$

is extremal.

P r o o f. The proof will be divided into three steps.

(1) First, we will show that $\left|p^{\prime}(z)\right| \leq\left\|T_{n}^{\prime}\right\|$ for every $z \in E_{1}$ such that

$$
|z| \leq|a \cos (\pi /(2 n))+i b \sin (\pi /(2 n))| .
$$

Consider the trigonometric polynomial $\tau_{n}(t)=p_{n}(a \cos t+i b \sin t)$. By the chain rule,

$$
\left|\tau_{n}^{\prime}(t)\right|==\left|p_{n}^{\prime}(z)\right| \cdot|-a \sin t+i b \cos t|, \quad z=a \cos t+i b \sin t .
$$

By Bernstein's inequality for trigonometric polynomials [7; 8, p. 216, Theorem 1] and (1.4),

$$
\left|p_{n}^{\prime}(z)\right|=\frac{\left|\tau_{n}^{\prime}(t)\right|}{|-a \sin t+i b \cos t|} \leq \frac{n\left\|p_{n}\right\|_{E_{1}}}{|-a \sin t+i b \cos t|} \leq \frac{n\left\|p_{n}\right\|_{E_{1}}}{\left|1-z^{2}\right|^{1 / 2}} .
$$

To estimate the numerator, we apply the well-known inequality [4, p. 240]

$$
\left\|p_{n}\right\|_{E_{1}} \leq \frac{(a+b)^{n}+(a-b)^{n}}{2}, \quad p_{n} \in \mathcal{P}_{n}^{[-1,1]} .
$$

Therefore,

$$
\left|p_{n}^{\prime}(z)\right| \leq \frac{n\left\|p_{n}\right\|_{E_{1}}}{|-a \sin t+i b \cos t|} \leq \frac{n\left((a+b)^{n}+(a-b)^{n}\right)}{2|-a \sin t+i b \cos t|}
$$


The image of the Chebyshev polynomial of the first kind $T_{n}(z)$ for $z \in E_{1}$ is the ellipse

$$
\left\{z=\frac{(a+b)^{n}+(a-b)^{n}}{2} \cos (n t)+i \frac{(a+b)^{n}-(a-b)^{n}}{2} \sin (n t) \mid t \in[0,2 \pi]\right\} .
$$

Hence,

$$
\left|T_{n}^{\prime}(z)\right|=\frac{n\left|-\left((a+b)^{n}+(a-b)^{n}\right) / 2 \cdot \sin (n t)+i\left((a+b)^{n}-(a-b)^{n}\right) / 2 \cdot \cos (n t)\right|}{|-a \sin t+i b \cos t|} .
$$

Let $z^{*}=a \cos (\pi /(2 n))+i b \sin (\pi /(2 n))$. Note that, if $|z|=|a \cos t+i b \sin t| \leq\left|z^{*}\right|$, then

$$
|i a \sin t+b \cos t| \geq|i a \sin (\pi /(2 n))+b \cos (\pi /(2 n))| .
$$

Therefore, for every $z \in E_{1}$ such that $|z| \leq\left|z^{*}\right|$, we have

$$
\left|T_{n}^{\prime}\left(z^{*}\right)\right|=\frac{\left.n\left((a+b)^{n}+(a-b)^{n}\right)\right)}{2|i a \sin (\pi /(2 n))+b \cos (\pi /(2 n))|} \geq\left|p_{n}^{\prime}(z)\right| .
$$

Now it is clear that the following inequality holds for $z$ such that $|z| \leq\left|z^{*}\right|$ :

$$
\left|p_{n}^{\prime}(z)\right| \leq\left\|T_{n}^{\prime}\right\|_{E_{1}} .
$$

(2) Let us obtain the estimate for $z \in E_{1}$ such that $|z|>\left|z^{*}\right|$. The idea of the proof of this point belongs to Erdös [5, Theorem 7].

Since $a^{2} \geq 1$, it is clear that

$$
\left|z^{*}\right|=\sqrt{a^{2} \cos ^{2} \frac{\pi}{2 n}+b^{2} \sin ^{2} \frac{\pi}{2 n}} \geq \cos \frac{\pi}{2 n} .
$$

Further, note that $\cos (\pi /(2 n))$ is the largest zero of the Chebyshev polynomial of the first kind.

Let us write the interpolation formula for $p_{n}^{\prime}(z)$ with roots of the Chebyshev polynomial as interpolation nodes. Denote them by $x_{k}, k=1, \ldots, n$. We have

$$
\left|p_{n}^{\prime}(z)\right|=\left|\sum_{k=1}^{n} \frac{T_{n}(z)}{\left(z-x_{k}\right)} \frac{p_{n}^{\prime}\left(x_{k}\right)}{T_{n}^{\prime}\left(x_{k}\right)}\right|=\left|T_{n}(z) \sum_{k=1}^{n} \frac{p_{n}^{\prime}\left(x_{k}\right)}{T_{n}^{\prime}\left(x_{k}\right)} \frac{\bar{z}-x_{k}}{\left|z-x_{k}\right|^{2}}\right|
$$

Note that an angle between any two vectors $\bar{z}-x_{k}$ is acute for $|z|>\cos (\pi /(2 n))$. Hence, if all the numbers $p_{n}^{\prime}\left(x_{k}\right) / T_{n}^{\prime}\left(x_{k}\right)$ are non-negative and have maximum moduli, then $p_{n}$ maximizes this expression over the set $\mathcal{P}_{n}^{[-1,1]}$.

By Bernstein's inequality [7; 8, p. 216, Theorem 1],

$$
\left|p_{n}^{\prime}\left(x_{k}\right)\right| \leq n / \sqrt{1-x_{k}^{2}}=\left|T_{n}^{\prime}\left(x_{k}\right)\right|
$$

for any $p_{n} \in \mathcal{P}_{n}^{[-1,1]}$. Therefore, the following estimate is true for $p_{n} \in \mathcal{P}_{n}^{[-1,1]}$ and $|z|>\cos (\pi /(2 n))$ :

$$
\left|p_{n}^{\prime}(z)\right| \leq\left|T_{n}^{\prime}(z)\right| \leq\left\|T_{n}^{\prime}\right\|_{E_{1}} .
$$

(3) It remains to prove that

$$
\left\|T_{n}^{\prime}\right\|_{E_{1}}=\frac{n}{2 b}\left[(a+b)^{n}-(a-b)^{n}\right] .
$$


It is known [6, p. $785,22.12 .2,22.12 .3]$ that

$$
T_{n}^{\prime}(z)= \begin{cases}2 n \sum_{k=1, k / 2 \notin \mathbb{Z}}^{n-2} T_{n-k}(z)+n, & (n-1) / 2 \in \mathbb{Z}, \\ 2 n \sum_{k=1, k / 2 \notin \mathbb{Z}}^{n-1} T_{n-k}(z), & (n-1) / 2 \notin \mathbb{Z} .\end{cases}
$$

Now it is clear that $\left\|T_{n}^{\prime}\right\|_{E_{1}}=\left|T_{n}^{\prime}(a)\right|$. Using (2.5), we see that

$$
\left|T_{n}^{\prime}(a)\right|=\left\|T_{n}^{\prime}\right\|_{E_{1}}=\frac{n}{2 b}\left[(a+b)^{n}-(a-b)^{n}\right] .
$$

\section{Remark}

The inequality considered in Section 2 is related to Markov-Bernstein-type inequalities. In 1889, A.A. Markov proved [9] that, if $p_{n}$ is a polynomial of degree $n$, then

$$
\left\|p_{n}^{\prime}\right\|_{[-1,1]} \leq n^{2}\left\|p_{n}\right\|_{[-1,1]} .
$$

Moreover, the Chebyshev polynomial of the first kind $T_{n}$ is the unique extremal polynomial.

A natural generalization of this problem is to find the best constant $M$ in the following inequality:

$$
\left\|p_{n}^{\prime}\right\|_{E_{1}} \leq M\left\|p_{n}\right\|_{E_{1}} \text {. }
$$

It is easy to see that, if $n=1$, then $T_{1}$ is extremal, since it deviates least from zero on $E_{1}$. It can be shown that $T_{2}$ is also extremal for $n=2$. However, A.C. Schaeffer and G. Szegö showed in 1941 [10, p. 223-225] that the solution to this problem for $n \geq 5$ is not always provided by the Chebyshev polynomial of the first kind. At present, this problem has not been solved.

\section{Acknowledgements}

The author is grateful to Dr. P. Yu. Glazyrina for constant attention to this work.

\section{REFERENCES}

1. Smirnov V. I., Lebedev N. A. Konstruktivnaya teoriya funkcij kompleksnogo peremennogo [The Constructive Theory of Functions of a Complex Variable]. Leningrad: Nauka Publ., 1964. 438 p. (in Russian)

2. Kolmogorov A.N. A remark on the polynomials of P.L. Chebyshev deviating the least from a given function. Uspehi Mat. Nauk, 1948. Vol. 3, No. 1. P. 216-221. (in Russian)

3. Kemperman J. H. B. Markov type inequalities for the derivatives of a polynomial. Aspects of Mathematics and its Applications, 1986. Vol. 34. P. 465-476. DOI: 10.1016/S0924-6509(09)70275-2

4. Duffin R., Schaeffer A.C. Some properties of functions of exponential type. Bull. Amer. Math. Soc., 1938. Vol. 4, No. 4. P. 236-240. DOI: 10.1090/S0002-9904-1938-06725-0

5. Erdös P. Some remark on polynomials. Bull. Amer. Math. Soc., 1947. Vol. 53, No. 12. P. 1169-1176. DOI: 10.1090/S0002-9904-1947-08938-2

6. Abramowitz M., Stegun I. A. Handbook of Mathematical Functions with Formulas, Graphs, and Mathematical Tables. NY: Dover Publications, 1965. 1046 p.

7. Bernstein S.N. O nailuchshem priblizhenii nepreryvnykh funktsii posredstvom mnogochlenov dannoi stepeni [On the Best Approximation of Continuous Functions by Polynomials of a Given Degree]. Comm. Soc. Math. Kharkov, 1912. 2 Series. Vol. XIII (13), No. 2-5. P. 49-194. (in Russian) https://www.math.technion.ac.il/hat/fpapers/bernstein1913.pdf 
8. Dzyadyk V.K. Vvedenie v teoriyu ravnomernogo priblizheniya funkcij polinomami [Introduction to the Theory of Uniform Approximation of Functions by Polynomials]. Moscow: Nauka, 1977. 508 p. (in Russian)

9. Markov A. A. Ob odnom voproce D.I. Mendeleeva [On a Question by D.I. Mendeleev]. Zap. Imp. Akad. Nauk., St. Petersburg, 1890. Vol. 62. P. 1-24. (in Russian)

10. Schaeffer A. C., Szegö G. Inequalities for harmonic polynomials in two and three dimensions. Trans. Amer. Math. Soc., 1941. Vol. 50. P. 187-225. DOI: 10.1090/S0002-9947-1941-0005164-7 